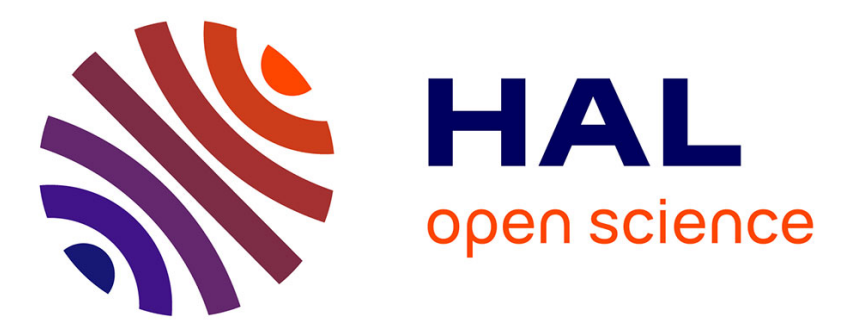

\title{
Molecular Sieves for the Separation of Hydrogen Isotopes
}

Julio Perez-Carbajo, José Parra, Conchi Maria Concepcion Ovin Ania, Patrick Merkling, Sofia Calero

\section{To cite this version:}

Julio Perez-Carbajo, José Parra, Conchi Maria Concepcion Ovin Ania, Patrick Merkling, Sofia Calero. Molecular Sieves for the Separation of Hydrogen Isotopes. ACS Applied Materials \& Interfaces, 2019, 11 (20), pp.18833-18840. 10.1021/acsami.9b02736 . hal-02124895

\section{HAL Id: hal-02124895 \\ https://hal.science/hal-02124895}

Submitted on 6 Nov 2020

HAL is a multi-disciplinary open access archive for the deposit and dissemination of scientific research documents, whether they are published or not. The documents may come from teaching and research institutions in France or abroad, or from public or private research centers.
L'archive ouverte pluridisciplinaire $\mathbf{H A L}$, est destinée au dépôt et à la diffusion de documents scientifiques de niveau recherche, publiés ou non, émanant des établissements d'enseignement et de recherche français ou étrangers, des laboratoires publics ou privés. 


\section{Perez-Carbajo J, Para JB, Ania CO, Merkling P, Calero S, Molecular Sieves for the Separation of Hydrogen Isotopes, ACS Applied Materials \& Interfaces 11 (2019) 18833-18840.}

\subsection{1/acsami.9b02736}

hal-02124895v1

\section{Molecular Sieves for the Separation of Hydrogen Isotopes}

Julio Perez-Carbajo

José B. Parra

Conchi O. Ania

Patrick J. Merkling

Sofia Calero

\section{Abstract}

Stable molecular hydrogen isotopes, $\mathrm{D}_{2}$ and $\mathrm{T}_{2}$, are both scarce and essential in several energy, industrial and large-scale, fundamental research applications. Due to the chemical similarity of these isotopes, their extraction and purification from hydrogen has relied for decades on expensive and energy-demanding processes. However, factoring in the phenomenon of quantum sieving could provide a new route for these separations. In this work, we have explored how to separate hydrogen isotopes by adsorption taking these quantum effects into account. To this end, we have conducted adsorption measurements to test our deuterium model, and performed a widespread computational screening over 210 pure-silica zeolites for $\mathrm{D}_{2}: \mathrm{H}_{2}$ and $\mathrm{T}_{2}: \mathrm{H}_{2}$ separations. Based on low-coverage adsorption properties, a reduced set of zeolites have been singled out and their performance in terms of adsorption capacity, selectivity and dynamic behavior have been assessed. Overall, the BCT-type zeolite clearly stands out for highly selective separations of both $\mathrm{D}_{2}$ and $\mathrm{T}_{2}$ over $\mathrm{H}_{2}$, achieving the highest reported selectivities at cryogenic temperatures. We also identified other interesting zeolites for the separation of hydrogen isotopes that offer an alternative way to tackle similar isotopic separations by an aimed selection or design of porous materials. 


\section{Introduction}

Separation of hydrogen isotopes is one of the most challenging current research areas, especially from an industrial point of view. The natural abundances of non-synthetic hydrogen isotopes are about $156 \mathrm{ppm}$ for deuterium (D) and only traces for tritium (T). Despite their scarcity, they both turn out to be crucial in several technological and industrial fields. Deuterium is employed in isotopic tracing for medical treatment and detection, nuclear magnetic resonance and neutron scattering techniques, and in development of deuterated drugs. Tritium has uses in the armament industry and in analytical chemistry. They are both used as raw materials in tokamak-type nuclear fusion reactors, where deuterium is also a key component for moderating neutrons. In fact, the yield of hydrogen isotopes for nuclear feedstock is under $10 \%$, so recovering them from waste is crucial to increase the efficiency and reduce nuclear residues. Additionally, even if hydrogen is the desired product, extraction of deuterium from hydrogen bulk might be economically attractive, while for the case of tritium its environmental impact may make this operation a mandatory requirement.

Chemical similarities between $\mathrm{H}_{2}, \mathrm{D}_{2}$, and $\mathrm{T}_{2}$ have been traditionally considered a major obstacle for the separation by molecular sieving methods. Other methods have therefore been used to carry out that separation: Cryogenic distillation, proton exchange reactions, thermal diffusion, centrifugation, electrolysis, or chromatography, among others. These techniques are quite energy consuming (the first three enumerated), or have a low yield (the last three mentioned). Thus, a cost-effective method with a high separation throughput would be desirable. In the mid-nineties, Beenakker et al. published a study on quantum sieving, an effect arising when the difference between the diameters of the pore and of the molecule approach the de Broglie wavelength. Under these conditions, similar molecules with different masses present different adsorption behaviors that can lead to heavier molecules in confined channels experiencing less repulsive interaction energies than lighter ones; this also affects diffusion properties in favor of the heavier isotope. That finding helped understanding the separation of isotopes in nanoporous materials. From then on, several works have explored hydrogen isotope separation in carbon nanotubes, zeolites, and metal-organic frameworks (MOFs). Although single-walled carbon nanotubes and zeolites were the first materials to be investigated, main research efforts in the last years have been focused in MOFs. Admitting that to exploit flexibility and breathing effects in MOFs is achieving promising results, zeolites, advantageously with respect to MOFs, are known for their thermal stability, economical production and scalability. In this sense, to the best of our knowledge, most studies on zeolites have only examined a few specific structures, so a widespread screening is lacking.

This work performs a computational investigation on 210 zeolites to find the best candidates for the separation of $\mathrm{D}_{2}$ or $\mathrm{T}_{2}$ from $\mathrm{H}_{2}$ at temperatures running from cryogenic up to $200 \mathrm{~K}$, and in the $10^{-1}-10^{5} \mathrm{kPa}$ pressure range. To that end, we have validated and used a Lennard-Jones (L-J) potential with Feynman-Hibbs quantum corrections for $\mathrm{H}_{2}$, and then derived the corresponding $\mathrm{L}-\mathrm{J}$ parameters for the isotopes. $\mathrm{D}_{2}$ interactions with pure silica zeolites have been validated with experimental adsorption isotherms at cryogenic temperatures. This study comprises an exhaustive comparison of hydrogen isotopes adsorption characteristics at low loadings, and then both dynamic and static analysis for selected zeolites to recommend the structures and working conditions leading to a highly-selective hydrogen isotope separation. 


\section{Methodology}

\section{Experimental procedures}

Experimental gas adsorption isotherms on pure silica zeolites (MFI and ITQ-29) at cryogenic temperatures were performed in a volumetric analyser provided with a turbomolecular vacuum pump and three pressure transducers $(0.13,1.33$ and $133 \mathrm{kPa}$, uncertainty within $0.15 \%$ of each reading). The volumetric analyser was coupled to a helium cryocooler (Gifford-McMahon) that allows a fine temperature control between $25-325 \mathrm{~K}$ with a stability of $\pm 0.1 \mathrm{~K}$. Isotherms were recorded in the pressure range between $10^{-2}$ and $120 \mathrm{kPa}$. Before the analysis, zeolites were outgassed under dynamic vacuum at $623 \mathrm{~K}(1 \mathrm{~K} / \mathrm{min})$ overnight. All gases were supplied by Air Products at an ultrahigh purity (i.e., 99.995\%). MFI and ITQ-29 (LTA topology) pure silica zeolites were supplied by the Institute of Chemical Technology (ITQ), being both structures nearly completely pure $\mathrm{SiO}_{2}$ crystals.

\section{Computational methods, models, and force field}

Energies between adsorbates themselves and with zeolites are dominated by electrostatic and van der Waals interactions. Electrostatic interactions are modeled by Coulombic potentials, using the Ewald summation to compute the long-range terms. On the other hand, Lennard-Jones (L-J) potentials are used to describe the van der Waals interactions. Quantum corrections have been added to Lennard-Jones potentials via an effective potential based on the Feynman-Hibbs variational approach to reproduce the quantum behavior of hydrogen isotopes at cryogenic temperatures. This approach has been repeatedly reported as an effective way to study the adsorption of quantum $\mathrm{H}_{2}, \mathrm{D}_{2}$, and $\mathrm{T}_{2}$ in zeolites and other nanoporous materials. Lennard-Jones potential with Feynman-Hibbs corrections is cut and shifted to zero at a cutoff radius of $12 \AA$.

Zeolite lattices are considered rigid and only formed by interconnected $\mathrm{SiO}_{4}$ tetrahedra. The coordinates of the framework atoms are taken from IZA database except for ISW, ITE, ITW, LTA, and MWW whose pure silica atom coordinates have been provided by ITQ: ITQ-7, ITQ-3, ITQ-12, ITQ-29, and ITQ-1, respectively. Static point charges for all of them $\left(\mathrm{q}_{\mathrm{Si}}=+0.786 \mathrm{e}^{-}\right.$ and $\left.\mathrm{q}_{\mathrm{O}}=-0.393 \mathrm{e}^{-}\right)$stem from Garcia-Sanchez et al.. Lennard-Jones interactions between framework atoms need not be considered in a rigid framework.

Hydrogen is modeled as a single, uncharged Lennard-Jones center, taken from van den Berg et $a l$, and modified by Deeg et al. to make it a Feynman-Hibbs potential. The Feynman-Hibbs parameters defining the interaction with zeolites have also been developed by Deeg. et al. Likewise, deuterium and tritium inherit the same model characteristics but updating their mass in relation to hydrogen molecule, so self- and cross-interaction Lennard-Jones parameters remain unaltered for them. Molecular weight of hydrogen isotopes are taken from NIST: $\mathrm{M}_{\mathrm{H} 2}=2.01588$ $u, \mathrm{M}_{\mathrm{D} 2}=4.02820 u$, and $\mathrm{M}_{\mathrm{T} 2}=6.03209 u$. These increases in mass directly affect the effective radius of the molecules as a consequence of the Feynman-Hibbs effective potential expression:

$$
U_{\mathrm{F}-\mathrm{H}}\left(r_{i j}\right)=U_{\mathrm{L}-\mathrm{J}}\left(r_{i j}\right)+\frac{\hbar^{2}}{24 \mu_{i j} k_{B} T}\left[U_{\mathrm{L}-\mathrm{J}}^{\prime \prime}\left(r_{i j}\right)+\frac{2 U_{\mathrm{L}-\mathrm{J}}^{\prime}\left(r_{i j}\right)}{r_{i j}}\right]
$$

where $\$ U_{-}\{\operatorname{|rm}\{\mathrm{L}-\mathrm{J}\}\} \$$ is the classical Lennard-Jones potential, prime and double prime 
symbols refer to first- and second-derivative, $r_{i j}$ is the distance between two interacting particles, $\mu_{i j}$ is the reduced mass of $i$ and $j$ particles given by $\mu_{i j}^{-1}=M_{i}^{-1}+M_{j}^{-1}$ (being $M$ the molecular mass), $\hbar$ is the reduced Planck constant, $k_{B}$ is the Boltzmann constant, and $T$ is the temperature.

Widom test-particle method, through Monte Carlo simulations using the so called Canonical ensemble, was used to determine Henry coefficients $\left(\mathrm{K}_{\mathrm{H}}\right)$ of adsorbates from $25 \mathrm{~K}$ to $200 \mathrm{~K}$. $\mathrm{K}_{\mathrm{H}}$ is related to the excess free energy of an adsorbed molecule by the following expression:

$$
K_{\mathrm{H}}=\frac{1}{\operatorname{RT} \rho_{\mathrm{f}}} \frac{\langle W\rangle}{\left\langle W^{i g}\right\rangle}
$$

where $T$ is the temperature, $R$ the gas constant, $\rho_{f}$ the density of the framework, $\langle W\rangle$ the Rosenbluth factor of the single chain molecule and $\left\langle W^{i g}\right\rangle$ the Rosenbluth factor of the molecule in the ideal gas. Given that $\mathrm{K}_{\mathrm{H}}$ provides information about adsorption at infinite dilution, selectivity at zero loading, $\mathrm{S}_{\mathrm{ij}}^{0}=\mathrm{K}_{\mathrm{H}}^{\mathrm{i}} / \mathrm{K}_{\mathrm{H}}^{\mathrm{j}}$, establishes a measure of preferential adsorption of adsorbate $i$ over $j$.

Adsorption isotherms were computed by Monte Carlo simulations in the Grand Canonical ensemble $(\mu V T)$, in which the chemical potential $\mu$ is directly related to fugacity and thereby to the pressure through the fugacity coefficient. Constant values were set for temperature and pressure in simulations, spanning the ranges $\mathrm{T}$ [25-200] K and, at least, $\mathrm{P}\left[10^{-1}-10^{5}\right] \mathrm{kPa}$. Both pure component and 1:1 mixture simulations were carried out for selected zeolites. Adsorption selectivity $\left(S_{i j}\right)$ highlights the preferential adsorption of one component $(i)$ over another $(j)$ from their molar fractions in the adsorbed phase $(\theta)$ and in the bulk phase $(x)$, according to:

$$
S_{i j}=\frac{\theta_{i} \cdot x_{j}}{\theta_{j} \cdot x_{i}}
$$

Molecular dynamics (MD) simulations were performed to obtain self-diffusion coefficients $(D)$ of the adsorbates. They were determined from the slope of the mean-square displacements once the adsorbed species reached the diffusive regime inside the structure. MD simulations were performed in the Canonical ensemble (NVT), integrating Newton's laws of motion using a velocity-Verlet algorithm with an integration time step of $0.5 \mathrm{fs}$ and simulated times upwards of 325 ns. A Nosé-Hoover chain thermostat was used to ensure the average temperature was constant. The number of adsorbates was set to 1 .

All the aforementioned techniques were simulated using RASPA molecular simulation software.

\section{Results and discussion}

\section{Deuterium/Hydrogen separation}

To determine the operational properties and performance of 1:1 hydrogen isotope separation using zeolites, we have carried out a thorough study on both equilibrium and time-dependent conditions. To that end, molecular simulations have proven to provide deep physical insights from which macroscopic behavior of adsorbates is inferred for these confined systems. Additionally, simulation methods allow to span the operation conditions further than 
experiments, which are restricted by economical considerations and number of trials. However, accurate models and force fields are completely necessary to obtain useful simulation results to target further experimental research. Therefore, we first validated the hydrogen model taken from literature and also the derived model for deuterium used in this work by comparing the computed and experimental adsorption isotherms of two well-known pure silica zeolites (MFI and LTA) at $77.3 \mathrm{~K}$ and $90.2 \mathrm{~K}$.
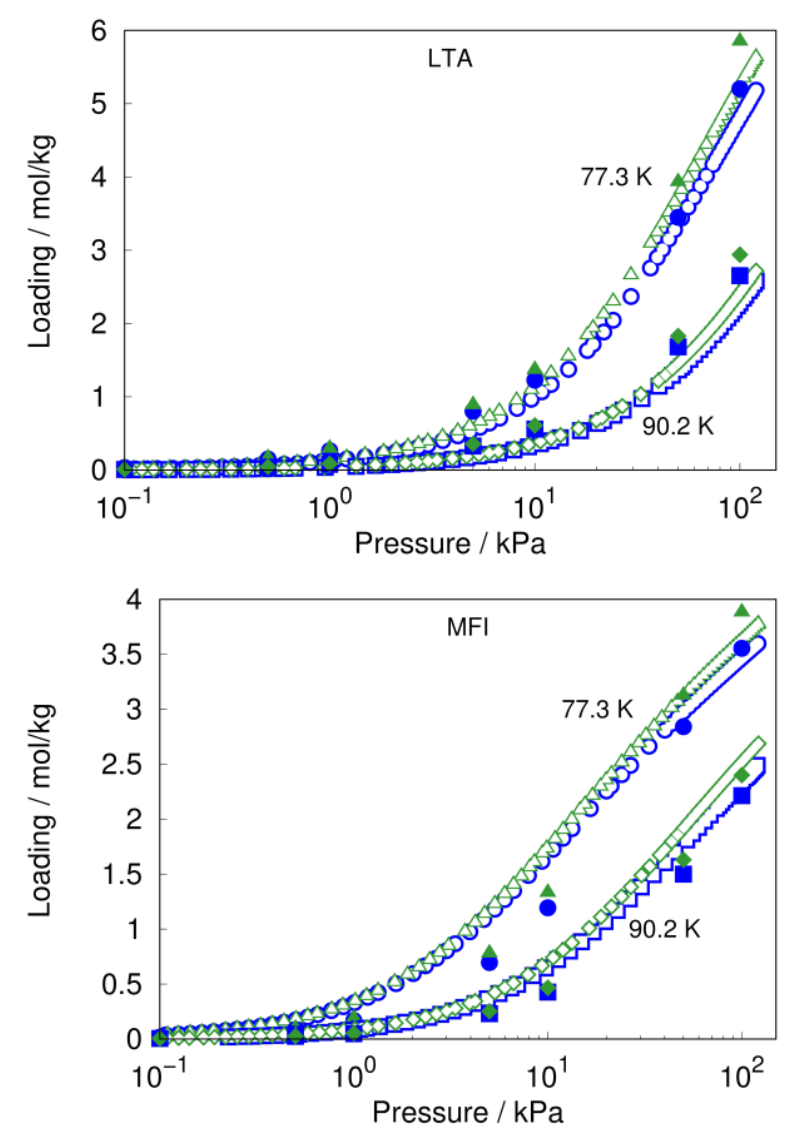

Figure 1: Experimental (open symbols) and simulated (filled symbols) adsorption isotherms of $\mathrm{D}_{2}$ (green triangles and diamonds) and $\mathrm{H}_{2}$ (blue circles and squares) in LTA (top) and MFI (bottom) at $77.3 \mathrm{~K}$ and $90.2 \mathrm{~K}$.

Both models reproduce precisely the experimental adsorption isotherms in LTA throughout the whole pressure range, as shown in Figure 1. Computed adsorption isotherms also reproduce the experimental data in MFI, although the loading of the two molecules is slightly underestimated at $77.3 \mathrm{~K}$ and 5-10 $\mathrm{kPa}$. Nevertheless, deviations affect both hydrogen and deuterium in much the same way, meaning selectivity determinations are reliable, and differences are not substantial either.

We have computed and analyzed Henry coefficients for $\mathrm{H}_{2}$ and $\mathrm{D}_{2}$ as a function of temperature in the range of $25-200 \mathrm{~K}$ for 210 zeolites. As a general trend, $\mathrm{K}_{\mathrm{H}}{ }^{\mathrm{D} 2}$ and $\mathrm{K}_{\mathrm{H}}{ }^{\mathrm{H} 2}$ are found to decrease with temperature and the heavier $\mathrm{D}_{2}$ is more strongly adsorbed than $\mathrm{H}_{2}$, therefore 
$\mathrm{S}_{\mathrm{D} 2 \mathrm{H} 2}^{0}>1$. This trend is declining as temperature increases (Figure 2). Figure 2 shows the five most selective zeolites at each temperature; as seen, zeolite BCT stands out by far as the best candidate (i.e., highest $S^{0}{ }_{\mathrm{D} 2 \mathrm{H} 2}$ ) to perform a separation at low loading over the whole temperature range. It is especially selective up to $50-60 \mathrm{~K}$. The second most selective zeolite depended on the temperature: AVL up to $50 \mathrm{~K}$ and MVY from 60-200K. Other zeolite structures in the top five at some temperatures are: AHT, ANA, BPH, EUO, IWW, LTJ, MSE, NSI, OSI, SAO, SBS, SBT, SSF, and SZR. All the computed $\mathrm{S}_{\mathrm{D} 2 \mathrm{H} 2}$ values are summarized in Table S1 in the Supporting Information.
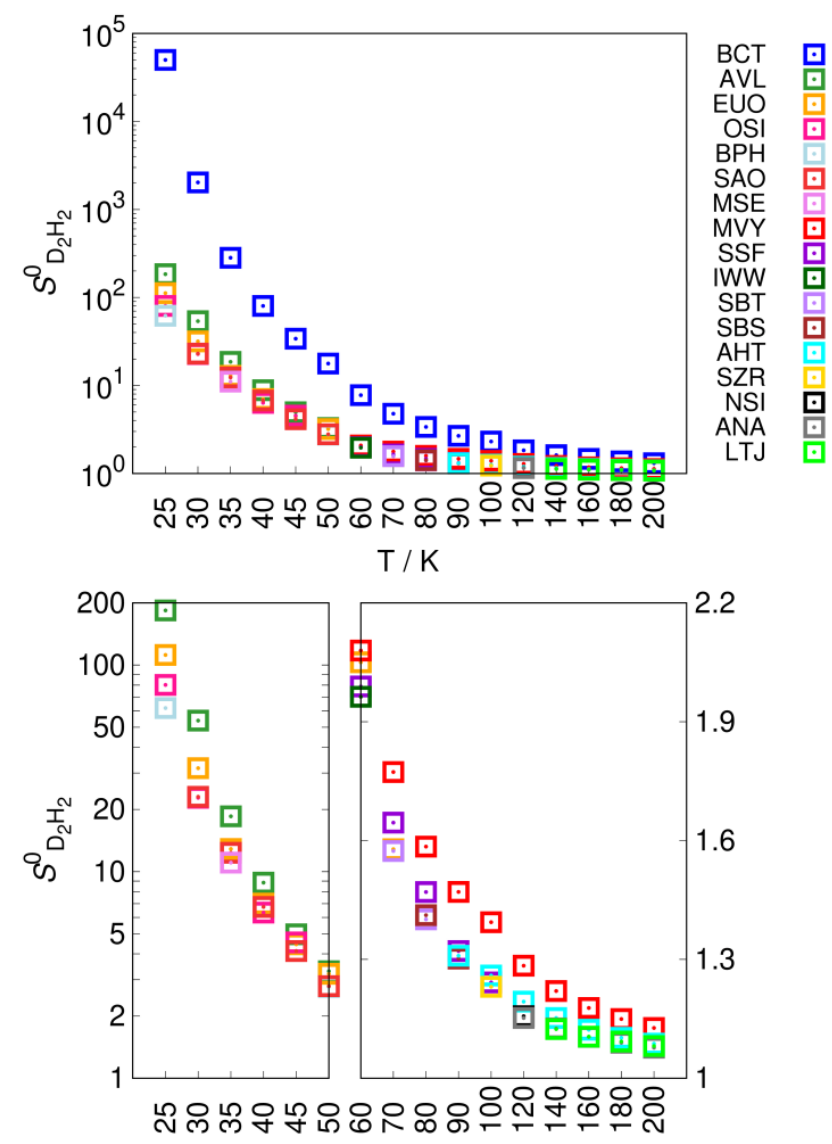

Figure 2: Selectivity at zero loading of deuterium over hydrogen as a function of temperature. Detailed graphics show close-up for the 25-50 K (bottom-left) and 60-200 K (bottom-right) temperature ranges, excluding $B C T$ zeolite to ease the view.

Given the superior selectivity values of zeolites BCT, AVL, and MVY for the separation of deuterium from hydrogen, these zeolites were investigated in more detail. Zeolite BCT has a tetragonal structure with a one-dimensional pore system, formed by parallel 8-membered-ring channels along the $z$-axial direction with transversal side-pockets on alternating sides. The orthorhombic form of zeolite MVY also has a one-dimensional, sinusoidal channel system, comprised of 10-membered-ring cavities along the $x$-axial direction. On the other hand, zeolite AVL is a two-dimensional structure, with a trigonal crystalline system consisting of cylindricallike cages connected by six 8-membered-ring windows (three on each end of the cylinder) and 
lentil-like cages with three 8-membered-ring windows (Figure 3).
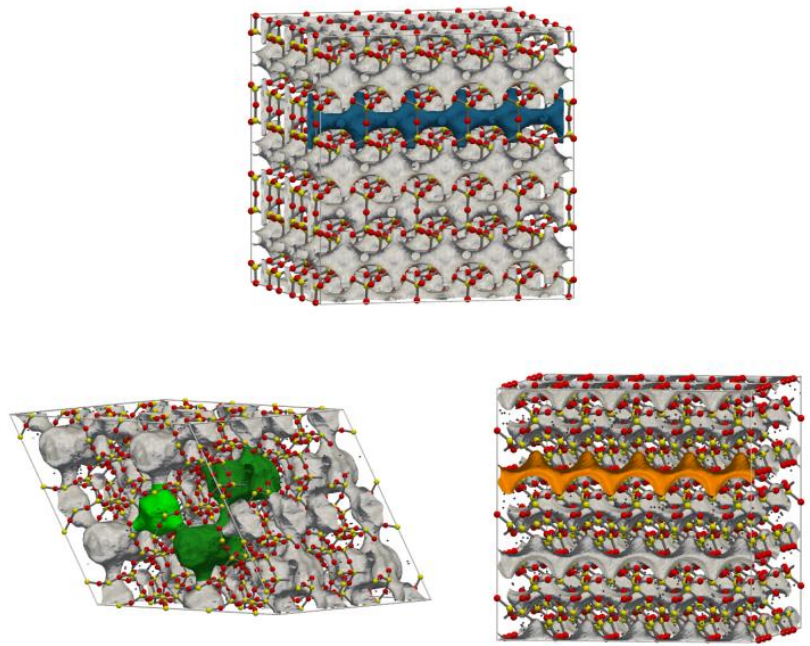

Figure 3: Energy surface areas and lattice atoms (yellow for Si and red for O) for BCT (top), AVL (bottom-left), and MVY (bottom-right) zeolites. Individual channels or pore systems are highlighted in blue, green, and orange, respectively, to ease the view. In AVL, bright-green is for lentil-like cages and dark-green for cylindrical cages.

Diffusion coefficients for single molecules have been evaluated with a twofold goal: determine the capacity of adsorbates to access adsorption centers inside zeolites and search for conditions under which a noticeable difference between $\mathrm{D}_{\mathrm{D} 2}$ and $\mathrm{D}_{\mathrm{H} 2}$ is reached, which would open up the possibility of performing a quantum kinetic sieving of the isotopes. Results are presented in Figure 4. Diffusion was computed in the temperature ranges in which zero-loading selectivities stood out: The whole range for BCT, 25-50 K for AVL, and 60-200 K for MVY. However, it is worth noting that diffusion coefficients for zeolite MVY at 80, 70, and $60 \mathrm{~K}$ could not be accurately determined after simulating $500 \mathrm{~ns}$ molecular dynamics, and were of no practical use. For the same reason, diffusion coefficients in BCT are not considered below $40 \mathrm{~K}$. Regarding kinetic sieving, diffusion coefficients for $\mathrm{D}_{2}$ and $\mathrm{H}_{2}$ are quite similar in AVL and MVY, and also for most of the analyzed temperatures in BCT. Although diffusion coefficients are quite low for zeolite BCT, a priori a kinetic-based separation at 40-50 K would be possible. 


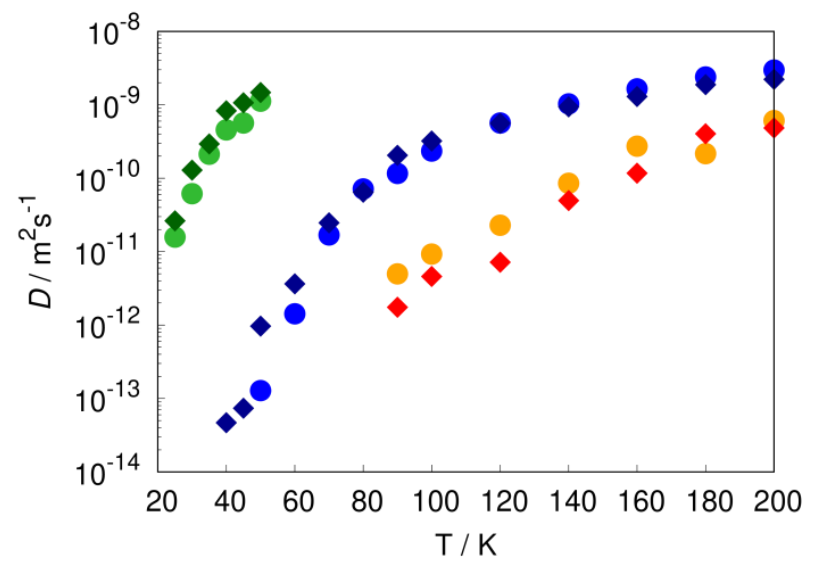

Figure 4: Self-diffusion coefficients of $\mathrm{D}_{2}$ (diamonds) and $\mathrm{H}_{2}$ (circles) in BCT (blue tones), $A V L$ (green tones), and MVY (orangy-red tones) at the temperatures at which they have high selectivity (see text) at zero loading.

After considering diffusion results, acceptable operation ranges to perform the $\mathrm{D}_{2} / \mathrm{H}_{2}$ separation were restricted to $25-50 \mathrm{~K}$ in AVL, 40-200 K in BCT, and 90-200 K in MVY. Pure compound as well as 1:1 mixture adsorption isotherms were simulated at the temperatures of choice spanning a pressure range of at least $\left[10^{-1}-10^{4}\right] \mathrm{kPa}$. For all three structures, in pure component adsorption isotherms (Figure S1), deuterium starts to be adsorbed at lower pressure than hydrogen, although this difference diminishes when temperature increases. Additionally, the location of the adsorption sites within the pores of the zeolites is shown for pure component adsorptions in Figures S2-S4 for BCT, AVL and MVY, respectively. Both deuterium and hydrogen adsorb at the same sites, and their loading dependency is also similar due to the competition for the same sites. Paradoxically, adsorbates' positions at high loading tend to be better defined as the adsorption sites are narrowed down by the steric interactions between adsorbates. The pure-component data suggest the separation capability of the zeolites but, rather than predicting mixture separation from pure compound isotherms as it is commonly reported in the literature, we simulated $1: 1 \quad \mathrm{D}_{2} / \mathrm{H}_{2}$ mixture adsorption isotherms to calculate $\mathrm{S}_{\mathrm{D} 2 \mathrm{H} 2}$ selectivities.

Figures 5, S5, and S6 contain the adsorption isotherms of the components of the mixture and also the temperature- and pressure-dependent adsorption selectivities in zeolites BCT, AVL, and MVY, respectively. In view of the adsorption selectivities exhibited by BCT (Figure 5, bottom), it must be noted that they are nearly parallel (in the log-log scale) over the whole pressure range at different temperatures. Additionally, the pressure dependence is rather small, selectivities remain within $0.8-1.3$ times the selectivity at $100 \mathrm{kPa}$, so that an increase in pressure to ensure a high loading is not detrimental to separation. Selectivities between 80 and 128 depending on pressure are reached at $40 \mathrm{~K}$, which, to the best of our knowledge, are the highest reported in the literature. It is especially advisable to maintain the pressure at $100 \mathrm{kPa}$ or more to obtain deuterium loadings of $3 \mathrm{~mol} / \mathrm{kg}$ or more. Under these cryogenic conditions, temperature control is important: a $20 \mathrm{~K}$ increase leads to selectivity decreases by one order of magnitude. Still, BCT achieves relevant separation ratios of $\sim 8-9$ at $60 \mathrm{~K}$. Further rises in temperature lead to more 
moderate decreases in selectivity, but then the absolute values are not very high.
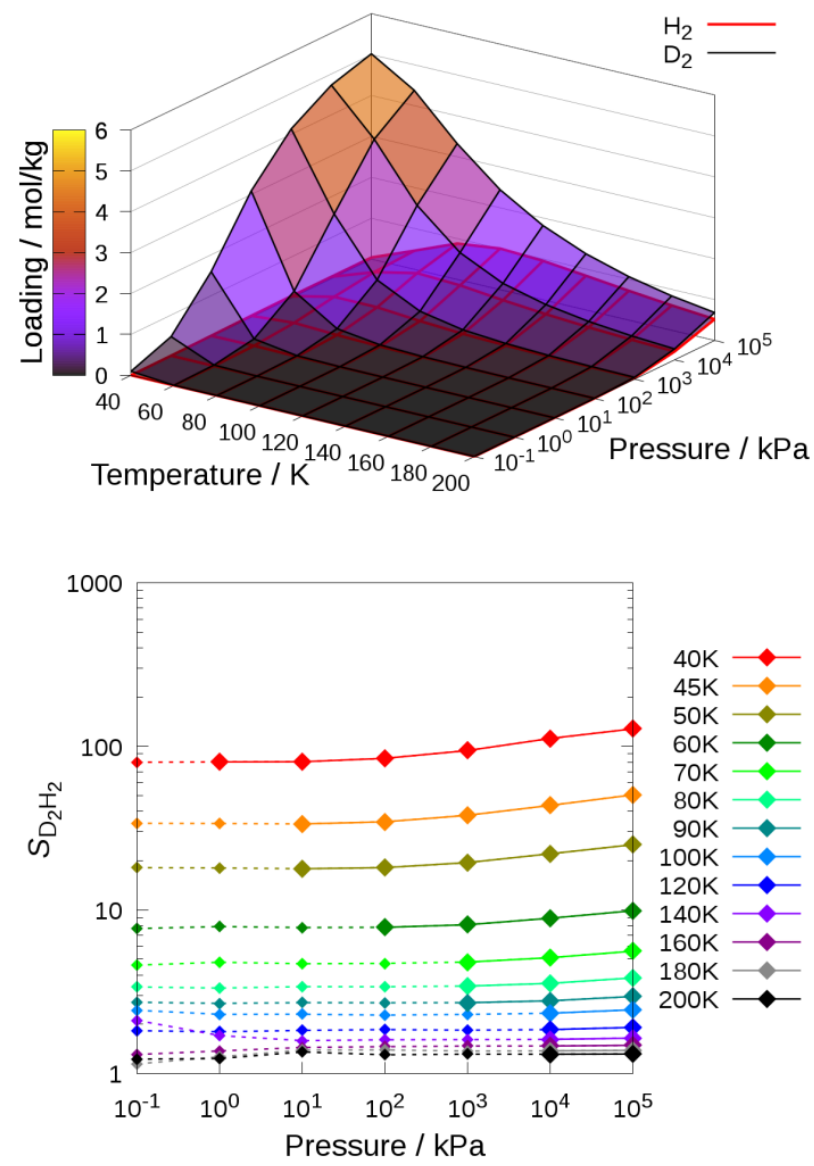

Figure 5: Adsorption loading (top) of deuterium (black lines grid) and hydrogen (red lines grid) from a 1:1 mixture as a function of pressure and temperature; grid cell colors match loading color-box. Adsorption selectivity (bottom) of deuterium over hydrogen as a function of pressure and temperature (color code assigned univocally for each temperature). Dashed lines and small symbols apply to selectivities at which the associated loading of $D_{2}$ is less than $0.1 \mathrm{~mol} / \mathrm{kg}$.

Zeolite AVL also achieves high $\mathrm{D}_{2} / \mathrm{H}_{2}$ adsorption selectivities at low temperatures (Figure S5). As in zeolite BCT, selectivity decreases with temperature but, in this case, its increase with pressure is much larger, reaching a remarkable value of $\mathrm{S}_{\mathrm{D} 2 \mathrm{H} 2}=22$ at $25 \mathrm{~K}$ and $10^{5} \mathrm{kPa}$ while adsorbing $14 \mathrm{~mol} / \mathrm{kg}$ of molecular deuterium vs. barely $0.6 \mathrm{~mol} / \mathrm{kg}$ of molecular hydrogen. It is also worth noting that $\mathrm{S}_{\mathrm{D} 2 \mathrm{H} 2}=15$ at $25 \mathrm{~K}$ and atmospheric pressure, with $13 \mathrm{~mol} / \mathrm{kg}$ deuterium adsorbed. Regarding zeolite MVY, selectivities remain poor, $\mathrm{S}_{\mathrm{D} 2 \mathrm{H} 2}<2$, remaining even under 1.5 for most of the operation conditions considered acceptable (Figure S6)

\section{Tritium/Hydrogen separation}

Following the same methodology used for $\mathrm{D}_{2}$ and $\mathrm{H}_{2}, \mathrm{~T}_{2} / \mathrm{H}_{2}$ separation at low coverage has been 
screened. As seen in Figure 6, BCT zeolite clearly stands out from the rest of zeolites, exhibiting the largest selectivity at all the temperatures simulated. Zeolites EUO, SAO, and OSI are second best at some temperature within the $25-80 \mathrm{~K}$ temperature range, for which these adsorption selectivities remain greater or equal to two.
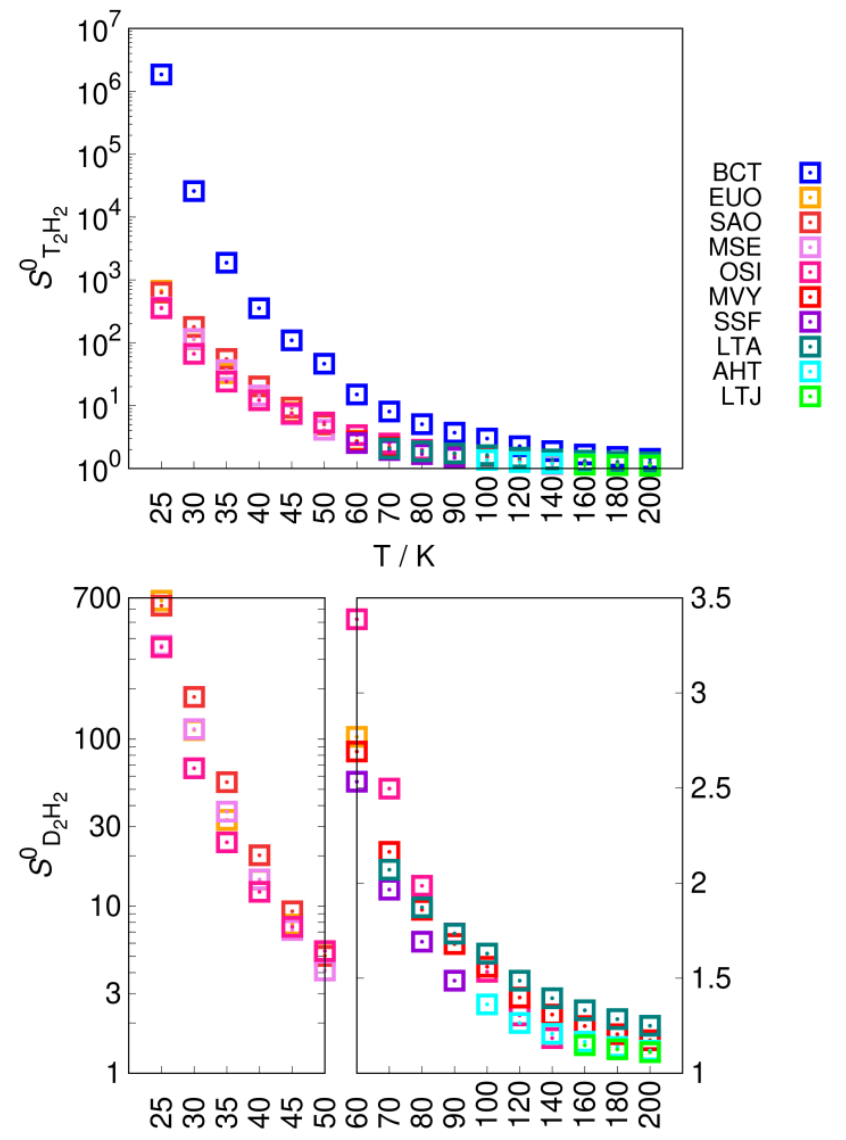

Figure 6: Selectivity at zero loading of tritium over hydrogen as a function of temperature. Detailed graphics show close-up for the 25-50 K (bottom-left) and 60-200 K (bottom-right) temperature ranges, excluding BCT zeolite to ease the view.

Given the prevalence of BCT for the separation of hydrogen isotopes, this zeolite was selected to further simulate the selectivity and adsorption performance for an equimolar $\mathrm{T}_{2} / \mathrm{H}_{2}$ mixture. Based on the diffusion coefficients obtained for $\mathrm{D}_{2}$ and $\mathrm{H}_{2}$, the adsorption of the equimolar mixture adsorption was computed at temperatures above $40 \mathrm{~K}$ (Figure 7). Although a similar shape of the adsorption isotherms/isobars is obtained compared with the deuterium case, it is worth noting that tritium loading is higher under the same (T,P) conditions while hydrogen is slightly less adsorbed, and therefore adsorption selectivity values rise up to $S_{\mathrm{T} 2 \mathrm{H} 2}=915$ under optimal conditions ( $\mathrm{T}=40 \mathrm{~K}$ and $\mathrm{P}=10^{5} \mathrm{kPa}$ ). 

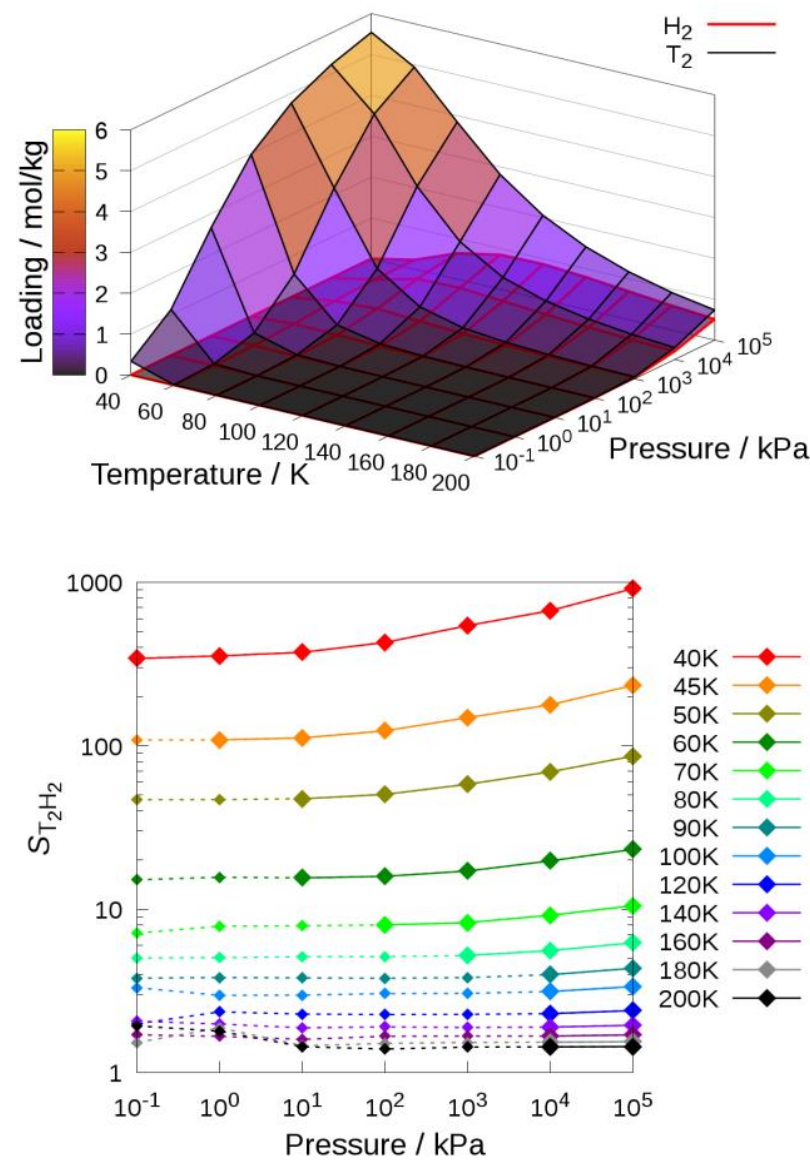

Figure 7: Adsorption loading (top) of tritium (black lines grid) and hydrogen (red lines grid) from a 1:1 mixture as a function of pressure and temperature; grid cell colors match loading color-box. Adsorption selectivity (bottom) of deuterium over hydrogen as a function of pressure and temperature (color code assigned univocally for each temperature). Dashed lines and small symbols apply to selectivities at which the associated loading of $T_{2}$ is less than $0.1 \mathrm{~mol} / \mathrm{kg}$.

\section{Conclusions}

We have screened various zeolite structures and operating conditions to select the best performing materials for the separation of hydrogen isotopes $\left(\mathrm{D}_{2} / \mathrm{H}_{2}\right.$ and $\mathrm{T}_{2} / \mathrm{H}_{2}$ mixtures). To this end, we have proposed a deuterium model developed from an existing hydrogen model that incorporates quantum corrections. The model has been validated vs experimental hydrogen and deuterium adsorption isotherms in two pure silica zeolites (MFI and LTA). At low temperatures, these quantum corrections are responsible for the stronger adsorption of deuterium over hydrogen, as expressed by the adsorption selectivity. The adsorption selectivity at low loadings was found to be a good indicator for the adsorption selectivity at higher loadings, and much more temperature-sensitive than pressure-dependent. We have identified several zeolites to perform hydrogen isotope separation with a high selectivity. Notably, zeolite BCT exhibits the highest adsorption selectivity for $\mathrm{D}_{2} / \mathrm{H}_{2}$ separation at low temperature. For instance, at $40 \mathrm{~K}$ and high 
loadings $\mathrm{D}_{2}$ is ca. 80 to 130 times more adsorbed than $\mathrm{H}_{2}$, depending on pressure. This zeolite also presents a high deuterium selectivity (ca. $\mathrm{S}_{\mathrm{D} 2 / \mathrm{H} 2}$ of 20 ) at $50 \mathrm{~K}$ and atmospheric pressure. We have also explored whether if in addition to the quantum sieving responsible for enhancing the adsorption of the heavier isotope, a quantum kinetic sieving effect -a diffusion-driven separation due to differences in the energy barriers- could be used advantageously in this system. Data showed that the quantum kinetic sieving effect was small in the temperatures of interest. Alternatively to BCT, zeolite AVL showed a high loading capacity between $25-45 \mathrm{~K}$, being able to separate deuterium from hydrogen reasonably well. Regarding $\mathrm{T}_{2} / \mathrm{H}_{2}$ separation, zeolite BCT has proven to have an extremely high selectivity, favoring the heavier isotope.

In sum, we have demonstrated that hydrogen isotope separation is feasible using zeolites at cryogenic temperatures and, especially, zeolite BCT stands out for this task. Besides, this study provides new perspectives to tackle other isotope mixture separations such as $\mathrm{H}_{2} / \mathrm{HD}$ (hydrogen deuteride) or $\mathrm{T}_{2} / \mathrm{D}_{2}$, by selecting or designing highly selective and efficient porous materials.

This work was supported by the European Research Council through an ERC Starting Grant (ERC2011-StG-279520-RASPA). We thank C3UPO for the HPC support.

Tables with adsorption selectivities for $\mathrm{D}_{2} / \mathrm{H}_{2}$ and $\mathrm{T}_{2} / \mathrm{H}_{2}$ are provided, as well as pure component adsorption isotherms and preferential sites of adsorption maps for $\mathrm{D}_{2}$ and $\mathrm{H}_{2}$ in $\mathrm{BCT}$, AVL and MVY zeolites.

\section{Supporting Information Available}

Tables with adsorption selectivities for $\mathrm{D}_{2} / \mathrm{H}_{2}$ and $\mathrm{T}_{2} / \mathrm{H}_{2}$ are provided, as well as pure component adsorption isotherms and preferential sites of adsorption maps for $\mathrm{D}_{2}$ and $\mathrm{H}_{2}$ in BCT, AVL and MVY zeolites.

\section{References}

(1) Greenwood, N.; Earnshaw, A. Chemistry of the Elements 2nd Edition; ButterworthHeinemann, 1997.

(2) Meija, J.; Coplen, T. B.; Berglund, M.; Brand, W. A.; De Bi_evre, P.; Gr $\square$ oning, M.;Holden, N. E.; Irrgeher, J.; Loss, R. D.; Walczyk, T.; Prohaska, T. IsotopicCompositions of the Elements 2013 (IUPAC Technical Report). Pure Appl. Chem.2016, 88, 293\{306.

(3) Cai, J.; Xing, Y.; Zhao, X. Quantum sieving: feasibility and challenges for theseparation of hydrogen isotopes in nanoporous materials. RSC Advances 2012, 2, 8579\{8586.

(4) Beenakker, J.; Borman, V.; Krylov, S. Y. Molecular Transport in Subnanometer ores:Zero-Point Energy, Reduced Dimensionality and Quantum Sieving. Chem. Phys. Lett.1995, 232, 379\{382

(5) Wang, Q.; Challa, S. R.; Sholl, D. S.; Johnson, J. K. Quantum Sieving in Carbon Nanotubes and Zeolites. Phys. Rev. Lett. 1999, 82, 956\{959. 
(6) Dresselhaus, M.; Williams, K.; Eklund, P. Hydrogen Adsorption in Carbon Materials.MRS Bull. 1999, 24, 45\{50.

(7) Challa, S. R.; Sholl, D. S.; Johnson, J. K. Adsorption and Separation of Hydrogenlsotopes in Carbon Nanotubes: Multicomponent Grand Canonical Monte CarloSimulations. J. Chem. Phys. 2002, 116, 814\{824.

(8) Tanaka, H.; Kanoh, H.; Yudasaka, M.; lijima, S.; Kaneko, K. Quantum E_ects onHydrogen Isotope Adsorption on Single-Wall Carbon Nanohorns. J. Am. Chem. Soc.2005, 127, 7511\{7516.

(9) Kowalczyk, P.; Gauden, P. A.; Terzyk, A. P. Cryogenic Separation of Hydrogen Isotopesin Single-Walled Carbon and Boron-nitride Nanotubes: Insight into the Mechanism ofEquilibrium Quantum Sieving in Quasi-One-Dimensional Pores. J Phys Chem B 2008,112, 8275\{8284.

(10) Niimura, S.; Fujimori, T.; Minami, D.; Hattori, Y.; Abrams, L.; Corbin, D.; Hata, K.;Kaneko, K. Dynamic Quantum Molecular Sieving Separation of D2 from H2\{D2Mixture with Nanoporous Materials. J. Am. Chem. Soc. 2012, 134, $18483\{18486$.

(11) Kumar, A. A.; Jobic, H.; Bhatia, S. K. Quantum E_ects on Adsorption and Di_usionof Hydrogen and Deuterium in Microporous Materials. J. Phys. Chem. B 2006, 110,16666\{16671.

(12) Chu, X.-Z.; Cheng, Z.-P.; Xiang, X.-X.; Xu, J.-M.; Zhao, Y.-J.; Zhang, W.-G.; Lv, J.S.; Zhou, Y.-P.; Zhou, L.; Moon, D.-K.; Lee, C.-H. Separation Dynamics of Hydrogenlsotope Gas in Mesoporous and Microporous Adsorbent Beds at $77 \mathrm{~K}$ : SBA15 andZeolites 5A, Y, 10x. Int. J. Hydrogen Energy 2014, 39, 4437\{4446.

(13) Kowalczyk, P.; Terzyk, A. P.; Gauden, P. A.; Furmaniak, S.; Pantatosaki, E.;Papadopoulos, G. K. Intrinsic D2/H2 Selectivity of NaX Zeolite: Interplay between Adsorption and Kinetic Factors. J. Phys. Chem. C 2015, 119, $15373\{15380$.

(14) Friebe, S.; Wang, N.; Diestel, L.; Liu, Y.; Schulz, A.; Mundstock, A.; Caro, J.Deuterium/Hydrogen Permeation through Di_erent Molecular Sieve Membranes: ZIF,LDH, Zeolite. Microporous Mesoporous Mater. 2015, 216, $127\{132$.

(15) Physick, A. J.; Wales, D. J.; Owens, S. H.; Shang, J.; Webley, P. A.; Mays, T. J.;Ting, V. P. Novel Low Energy Hydrogen\{Deuterium Isotope Breakthrough SeparationUsing a Trapdoor Zeolite. Chem. Eng. J. 2016, 288, $161\{168$.

(16) Salazar, J.; Lectez, S.; Gauvin, C.; Macaud, M.; Bellat, J.; Weber, G.; Bezverkhyy, I.;Simon, J. Adsorption of Hydrogen Isotopes in the Zeolite NaX: Experiments andSimulations. Int. J. Hydrogen Energy 2017, 42, 13099\{13110

(17) Pantatosaki, E.; Jobic, H.; Kolokolov, D. I.; Karmakar, S.; Biniwale, R.;Papadopoulos, G. K. Probing the Hydrogen Equilibrium and Kinetics in Zeolitelmidazolate Frameworks Via Molecular Dynamics and Quasi-elastic Neutron ScatteringExperiments. J. Chem. Phys. 2013, 138, 034706.

(18) FitzGerald, S. A.; Pierce, C. J.; Rowsell, J. L.; Bloch, E. D.; Mason, J. A. Highly 
Selective Quantum Sieving of D2 from H2 by a Metal\{Organic Framework AsDetermined by Gas Manometry and Infrared Spectroscopy. J. Am. Chem. Soc. 2013,135, 9458\{9464.

(19) Oh, H.; Savchenko, I.; Mavrandonakis, A.; Heine, T.; Hirscher, M. Highly E_ectiveHydrogen Isotope Separation in Nanoporous Metal\{Organic rameworks with OpenMetal Sites: Direct Measurement and Theoretical Analysis. ACS Nano 2014, 8, 761\{770.

(20) Weinrauch, I.; Savchenko, I.; Denysenko, D.; Souliou, S.; Kim, H.; Le Tacon, M.;Daemen, L. L.; Cheng, Y.; Mavrandonakis, A.; Ramirez-Cuesta, A.; Volkmer, D.;Sch $\square$ tz, G.; Hirscher, M.; Heine, T. Capture of Heavy Hydrogen Isotopes in a Metal-Organic Framework with Active Cu (I) Sites. Nat. Commun. 2017, 8, 14496.

(21) Kim, J. Y.; Balderas-Xicoht_encatl, R.; Zhang, L.; Kang, S. G.; Hirscher, M.; Oh, H.;Moon, H. R. Exploiting Di_usion Barrier and Chemical A_nity of

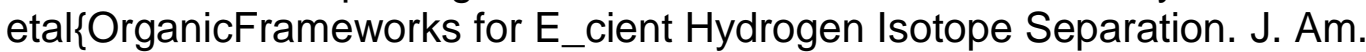
Chem. Soc. 2017, 139,15135\{15141.

(22) Feynman, R. P.; Hibbs, A. R. Quantum Mechanics and Path Integrals; McGrawHill, 1965.

(23) Ses_e, L. M. Study of the Feynman-Hibbs E_ective Potential against the PathIntegralFormalism for Monte Carlo Simulations of Quantum Many-Body Lennard-JonesSystems. Mol. Phys. 1994, 81, 1297\{1312.

(24) Ses_e, L. M. Feynman-Hibbs Potentials and Path Integrals for Quantum LennardJonesSystems: Theory and Monte Carlo Simulations. Mol. Phys. 1995, 85, $931\{947$.

(25) Darkrim, F.; Aou_, A.; Levesque, D. Quantum Contribution to Gas Adsorption inCarbon Nanotubes. Mol. Simul. 2000, 24, 51\{61.(26) Noguchi, D.; Tanaka, H.; Kondo, A.; Kajiro, H.; Noguchi, H.; Ohba, T.;Kanoh, H.; Kaneko, K. Quantum Sieving E_ect of Three-dimensional $\mathrm{Cu}$-Based OrganicFramework for $\mathrm{H} 2$ and D2. J. Am. Chem. Soc. 2008, 130, 6367\{6372.

(27) Tanaka, H.; Noguchi, D.; Yuzawa, A.; Kodaira, T.; Kanoh, H.; Kaneko, K. QuantumE_ects on Hydrogen Isotopes Adsorption in Nanopores. J. Low Temp. Phys. 2009, 157,352\{373.

(28) Noguchi, D.; Tanaka, H.; Fujimori, T.; Kagita, H.; Hattori, Y.; Honda, H.; Urita, K.;Utsumi, S.; Wang, Z.-M.; Ohba, T.; Kanoh, H.; Hata, K.; Kaneko, K. Selective D2Adsorption Enhanced by the Quantum Sieving E_ect on Entangled SingleWall Carbon Nanotubes. J. Phys.: Condens. Matter 2010, 22, 334207.

(29) Baerlocher, C.; McCusker, L.; Olson, D. Atlas of Zeolite Framework Types, 6th ed.;2007.

(30) Villaescusa, L. A.; Barrett, P. A.; Camblor, M. A. ITQ-7: A New Pure Silica Polymorph with a Three-Dimensional System of Large Pore Channels. Angew. Chem. Int. Ed.1999, 38, 1997\{2000.

(31) Camblor, M. A.; Corma, A.; Lightfoot, P.; Villaescusa, L. A.; Wright, P. A. Synthesis and Structure of ITQ-3, the First Pure Silica Polymorph with a Two-Dimensional 
System of Straight Eight-Ring Channels. Angew. Chem. Int. Ed. 1997, 36, $2659\{2661$.

(32) Barrett, P. A.; Boix, T.; Puche, M.; Olson, D. H.; Jordan, E.; Koller, H.; Camblor, M. A. ITQ-12: A New Microporous Silica Polymorph Potentially Useful for Light Hydrocarbon Separations. Chem. Commun. 2003, 2114\{2115.

(33) Corma, A.; Rey, F.; Rius, J.; Sabater, M. J.; Valencia, S. Supramolecular SelfAssembled Molecules As Organic Directing Agent for Synthesis of Zeolites. Nature2004, 431, 287.

(34) Camblor, M. A.; Corma, A.; D_az-Caba nas, M.-J.; Baerlocher, C. Synthesis andStructural Characterization of MWW Type Zeolite ITQ-1, the Pure Silica Analog ofMCM-22 and SSZ-25. J. Phys. Chem. B 1998, 102, 44\{51.

(35) Garcia-Sanchez, A.; Ania, C. O.; Parra, J. B.; Dubbeldam, D.; Vlugt, T. J. H.;Krishna, R.; Calero, S. Transferable Force Field for Carbon Dioxide Adsorption inZeolites. J. Phys. Chem. C 2009, 113, 8814\{8820.21

(36) van den Berg, A. W.; Bromley, S. T.; Wojdel, J. C.; Jansen, J. C. Adsorption Isothermsof H2 in Microporous Materials with the SOD Structure: A Grand Canonical MonteCarlo Study. Microporous Mesoporous Mater 2006, 87, $235\{242$.

(37) Deeg, K. S.; Guti_errez-Sevillano, J. J.; Rocio Bueno-Perez, J. B. P.; Ania, C. O.;Doblar_e, $\bar{M}_{\text {.; }}$ Calero, S. Insights on the Molecular Mechanisms of Hydrogen Adsorptionin Zeolites. J. Phys. Chem. C 2013, 114374\{14380.

(38) Stein, S. NIST Chemistry WebBook, NIST Standard Reference Database Number 69 ,Eds. PJ Linstrom and WG Mallard.

(39) Widom, B. Some Topics in the Theory of Fluids. J. Chem. Phys. 1963, 39, $2808\{2812$.

(40) Vlugt, T.; Garcia-Perez, E.; Dubbeldam, D.; Ban, S.; Calero, S. Computing the Heat ofAdsorption Using Molecular Simulations: The E_ect of Strong Coulombic Interactions.J. Chem. Theory Comput. 2008, 4, 1107\{1118.

(41) Dubbeldam, D.; Beerdsen, E.; Vlugt, T. J. H.; Smit, B. Molecular Simulation ofLoading-Dependent Di_usion in Nanoporous Materials Using Extended DynamicallyCorrected Transition State Theory. J. Chem. Phys. 2005, 122, \{.

(42) Hoover, W. G. Constant\{Pressure Equations of Motion. Phys. Rev. A 1986, 34, $2499\{2500$.

(43) Dubbeldam, D.; Calero, S.; Donald, E.; Snurr, R. RASPA: Molecular SimulationSoftware for Adsorption and Di_usion in Flexible Nanoporous Materials. Mol. Simul.2015,

(44) Dubbeldam, D.; Torres-Knoop, A.; Walton, K. S. On the Inner Workings of MonteCarlo Codes. Mol. Simul. 2013, 39, 1253\{1292.22 